\title{
3D-imaging: a scanning light pattern projector
}

Bøgh Stokholm, Mathias; Hanson, Steen Grüner; Kjær, Rasmus; Allin, Thomas Højgaard; Jakobsen, Michael Linde

\section{Published in:}

Applied Optics

Link to article, DOI:

10.1364/AO.55.009074

Publication date:

2016

Document Version

Peer reviewed version

Link back to DTU Orbit

Citation (APA):

Bøgh Stokholm, M., Hanson, S. G., Kjær, R., Allin, T. H., \& Jakobsen, M. L. (2016). 3D-imaging: a scanning light pattern projector. Applied Optics, 55(32), 9074-9083. https://doi.org/10.1364/AO.55.009074

\section{General rights}

Copyright and moral rights for the publications made accessible in the public portal are retained by the authors and/or other copyright owners and it is a condition of accessing publications that users recognise and abide by the legal requirements associated with these rights.

- Users may download and print one copy of any publication from the public portal for the purpose of private study or research.

- You may not further distribute the material or use it for any profit-making activity or commercial gain

- You may freely distribute the URL identifying the publication in the public portal

If you believe that this document breaches copyright please contact us providing details, and we will remove access to the work immediately and investigate your claim. 


\title{
3D-imaging: A scanning light pattern projector
}

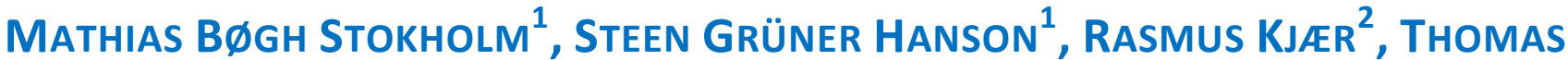 \\ ALLIN $^{2}$ AND MiChaEL LINDE JAKOBSEN ${ }^{1 *}$
}

\author{
${ }^{1}$ DTU Fotonik, Department of Photonics Engineering, Technical University of Denmark, P.O. Box 49, DK-4000, Roskilde, Denmark \\ ${ }^{2} 3$ Shape A/S, Holmens Kanal 7, DK-1060 Copenhagen, Denmark \\ *Corresponding author: $\underline{\text { mlja@fotonik.dtu.dk }}$
}

Received XX Month XXXX; revised XX Month, XXXX; accepted XX Month XXXX; posted XX Month XXXX (Doc. ID XXXXX); published XX Month XXXX

\begin{abstract}
The technology of electrically adjustable optical interfaces has found applications in e.g. camera lenses, where an adjustable focal length provides automatic focusing for the camera. In this paper we will investigate a liquid lens, where both the focal length and the tilt of this lens can be adjusted electrically. Specifically, the tilting ability of this lens will be tested by combining the liquid lens with a projector in order to scan lines across a 3D object. Linearity, reproducibility, hysteresis and time response of its tilting functionality will be tested. Further, cross-talk between the two functionalities of the liquid lens is tested for the specific case, where the focal length is set to infinity. Finally, the liquid lens and the projector in combination with four stereo cameras will be demonstrated as a 3D imaging setup. (C) 2016 Optical Society of America
\end{abstract}

OCIS codes: (220.2740) Geometric optical design; (110.2945) Illumination design; (230.2090) Electro-optical devices; (150.6910) Three-dimensional sensing; (110.6880) Three-dimensional image acquisition; (170.1850) Dentistry.

http://dx.doi.org/10.1364/AO.99.099999

\section{Introduction}

The area of 3D imaging is experiencing rapid growth. This is especially true for applications within computer vision, 3D shape measurements for e.g. 3D printing, automatic inspection and recognition issues. The demands for speed, precision, and low-price are high. In dentistry applications, casts of imprints of patient's teeth and surrounding tissue need to be measured precisely (within few microns) in order to construct prosthetics that fit tightly. The entire surface of such a cast needs to be measured without holes or occlusions. To overcome occlusions, edges and changes in surface reflectivity, objects are re-orientated and rescanned. This also increases data reliability, and allows for the capturing of most of the 360-deg shape of the object.

Various optical techniques are currently being developed, mostly driven by these demands. Time-of-flight methods measure the time of flight of either a pulsed or modulated light source directly. Typical depth resolutions for time-of-flight point measurements are in the range of a millimeter [1].

Triangulation [2] and stereo imaging [3] are alternative methods for $3 \mathrm{D}$ imaging. On the cost of speed, these methods provide depths and lateral position measurements with a resolution of several microns. A structured light pattern is projected onto the 3D object and one or several images are acquired of the object from different angles. A simple light structure such as a single line eases the processing of the images. However, the scanning time and the requirements to the mechanical scanner will be high. By projecting several lines onto the
3D object, the number of measurement points per image increases dramatically, and the scanning distance/time drops accordingly. However, without a coding [4-7] of the lines or other ways to distinguish between the individual lines, the correspondence problem will limit the density/number of lines on the 3D object. But, if the scanning range becomes sufficiently small other scanning methods, rather than the motor-based methods, can be considered.

Typically, scans of a structured light pattern are implemented using a high-precision mechanical stage, or display device, e.g. Liquid Crystal Display (LCD). The mechanical scanners are precise but consume relatively high amounts of power, emit electrical noise, cause mechanical vibrations and wear down over time. Of non-mechanical options, DLP projectors [6, 7] and MEMS mirrors [8] can be mentioned. However, mechanical scanners, displays and projectors are all expensive devices.

In this paper we will describe the use of a liquid lens (Baltic 617) from Varioptic [9]. This lens has an interface between two encapsulated and immiscible liquids. With the help of several electrical electrodes, the interface can be tilted for beam deflection and curved for autofocus applications. In our implementation, the liquid lens will be mounted as an extension to a projector lens used for projecting lines onto a 3D object. In this way, the image from the projector can be scanned across the 3D object. The principal behavior of the lens will be described using $3 \times 3$ ray-transfer matrices [10]. The performance of the scanning projector will be tested and, finally, the projector will be applied to a 3D imaging device.

\section{Principle of the liquid lens}


The Baltic 617 lens is based on a technology called Electro Wetting On Dielectric (EWOD) [11]. The electrodes are coated with a thin insulating dielectric layer, which isolates them electrically from a conductive liquid, present on the other side of the coating. By applying a positive electrical field to the electrodes relative to the conducting liquid, the electrodes will attract the conductive liquid [12].

The liquid lens has two transparent liquids of equal density but different refractive indices encapsulated between two glass windows. One of these liquids is the conductive liquid, while the other is nonconducting. The two liquids are immiscible, and will have a welldefined interface between them, due to surface tension. The insulated electrodes are placed on the circumference of the liquid interface in contact with the interface. Therefore, by applying an electric field to the electrodes relative to the conducting liquid, the circumference of the liquid interface will move along the surface of the electrodes in the direction normal to the circumference. When this happens, the constraints on constant liquid volumes will alter the radius of curvature of the interface between the two liquids $[11,13]$. The shape of the interface will be parabolic. The behavior of the interface is controlled by five insulated electrodes: One common (GND) and four independent electrodes $\left(V_{x+}, V_{x-}, V_{y+}, V_{y-}\right)$ as illustrated in Fig. 1a. By applying a common voltage $V_{A F}$ within the range from 20-70 $V_{\text {RMS }}$ to the four independent electrodes relative to the common electrode, the radius of curvature of the interface can be varied from $-3.0 \mathrm{~mm}$ to $-\infty$, through the discontinuity (a nearly flat interface) to $\infty$ and again continuously from $\infty$ to $3.8 \mathrm{~mm}$. By applying a differential voltage between various combinations of the four independent electrodes, tilt of the interface can be applied arbitrarily in the $x$ and $y$ directions $\left(\theta_{x}\right.$, $\theta_{\text {y) }}$, as described below [9]:

$$
\left(\begin{array}{l}
V_{x+} \\
V_{x-} \\
V_{y+} \\
V_{y-}
\end{array}\right)=V_{A F}\left(\begin{array}{l}
1 \\
1 \\
1 \\
1
\end{array}\right)+\left(\begin{array}{cc}
G_{\text {tilt }} & 0 \\
-G_{\text {tilt }} & 0 \\
0 & G_{\text {tilt }} \\
0 & -G_{\text {tilt }}
\end{array}\right)\left(\begin{array}{l}
\alpha_{x} \\
\alpha_{y}
\end{array}\right),
$$

where $G_{\text {tilt }}$ is constant with the units V/deg. and $\left(\alpha_{x}, \alpha_{y}\right)$ is the resulting light-ray deflection in deg., caused by the tilt of the interface.

We will describe the optical principle of the liquid lens and the optical system using $3 \times 3$ ray-transfer matrices [10]. The $3 \times 3$ raytransfer matrix is based on the paraxial-optical-ray condition and contains the same four elements as the traditional $\mathrm{ABCD}$ ray transfer matrix [14]. However, the $3 \times 3$ ray-transfer matrix has an additional row and column, which allow for information $(E, F)$ about tilt or/and off-set of the individual components:

$$
\left(\begin{array}{c}
y_{2} \\
\alpha_{2} \\
1
\end{array}\right)=\left(\begin{array}{lll}
A & B & E \\
C & D & F \\
0 & 0 & 1
\end{array}\right)\left(\begin{array}{c}
y_{1} \\
\alpha_{1} \\
1
\end{array}\right) .
$$

We assume that all optical components in the liquid lens, except the interface between the two liquids, are perfectly aligned both in terms of tilt and off-set ( $E=F=0$ ). Therefore, we divide our considerations into three paths; 1) The path from the front aperture to a position immediately before the liquid interface; 2 ) The path through the liquid interface. 3) The path from a position immediately after the liquid interface to the rear aperture. The apertures are described by the complex ABCD matrix [15], tacitly assuming that the aperture are Gaussian apodized. A theoretical description of a hard circular aperture can in terms of transmitted power be replaced successfully with a Gaussian apodized apertures by decreasing the radius of the Gaussian aperture by a factor of $2^{1 / 2}$ [16] relative to the radius of a hard aperture.
In Fig. $1 \mathrm{~b}$ the individual components of the liquid lens are illustrated. The first path starts in air at the front aperture to the left. The refractive index of air is $n_{a}$ and the opening radius of the aperture is $\sigma$ :

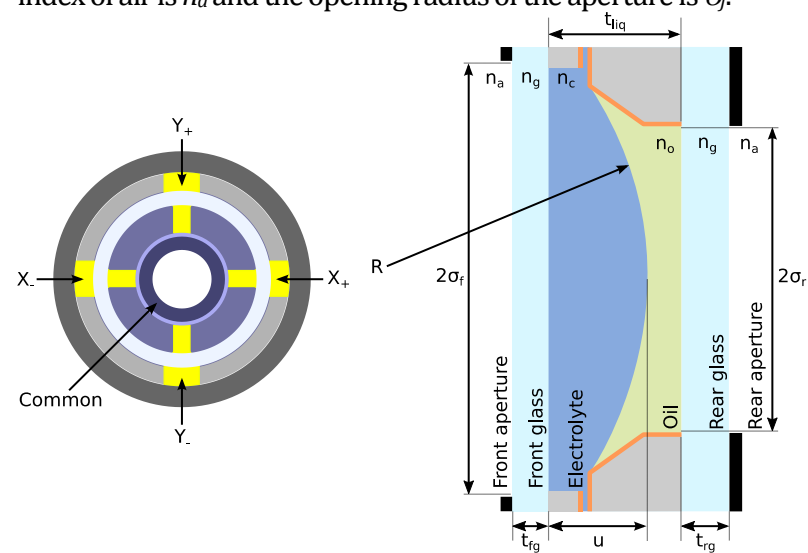

Fig. 1. The schematic illustrates (a) the five electrodes, which controls the liquid lens, and (b) the optical layout of the liquid lens (Varioptics).

Then, a ray will be refracted at the flat interface between air and the front glass window. The refractive index of the front glass window is $n_{g}$. After propagating a distance of $t_{f g}$ through the front glass window, a ray will be refracted at the flat interface between glass and the conductive liquid. The refractive index of the conductive liquid is $n_{c}$ and the final distance of propagation through the conductive liquid is $u$. The thickness $u$ of the conductive liquid phase is dependent of the common voltage $V_{A F}$, as it controls the curvature of the liquid interface. We find the following $3 \times 3$ ray-transfer matrix $\mathbf{T}_{\mathbf{f i}}$ for the first path:

$$
\mathbf{T}_{\mathrm{fi}}=\left(\begin{array}{ccc}
1-\frac{2 i n_{a}}{k \sigma_{f}^{2}} C_{o} & n_{a} C_{o} & 0 \\
-\frac{2 i n_{a}}{k n_{c} \sigma_{f}^{2}} & \frac{n_{a}}{n_{c}} & 0 \\
0 & 0 & 1
\end{array}\right),
$$

where

$$
C_{o}=\frac{u}{n_{c}}+\frac{t_{f g}}{n_{g}} .
$$

The third path starts immediately after the interface between the two liquids, and propagates a distance of $t_{\text {liq }}-u$ through the oil until refraction at the flat interface between the oil and the rear glass window. The refractive index of the oil and glass is $n_{o}$ and $n_{g}$, respectively. The thickness of the rear glass window is $t_{r g}$ and the radius of the rear aperture is $\sigma_{r}$. We find the following $3 \times 3$ ray-transfer matrix $\mathbf{T}_{\text {ir }}$ for the third path:

$$
\mathbf{T}_{\mathrm{ir}}=\left(\begin{array}{ccc}
1 & n_{o} C_{c} & 0 \\
-\frac{2 i}{k \sigma_{r}^{2}} & \frac{n_{o}}{n_{a}}-\frac{2 i n_{o}}{k \sigma_{r}^{2}} C_{c} & 0 \\
0 & 0 & 1
\end{array}\right),
$$

where

$$
C_{c}=\frac{t_{l i q}-u}{n_{o}}+\frac{t_{r g}}{n_{g}} .
$$




$$
\mathbf{T}_{\text {Comb }}\left(\theta_{y}\right)=\left(\begin{array}{ccc}
1 & f(1+|M|)-\Delta & 0 \\
0 & 1 & 0 \\
0 & 0 & 1
\end{array}\right) \mathbf{T}_{\text {Tot }}\left(\theta_{y}\right)\left(\begin{array}{ccc}
1 & 0 & 0 \\
-\frac{1}{f} & 1 & 0 \\
0 & 0 & 1
\end{array}\right)\left(\begin{array}{ccc}
1 & f\left(1+\frac{1}{|M|}\right) & 0 \\
0 & 1 & 0 \\
0 & 0 & 1 \\
&
\end{array}\right),
$$

According to the condition of paraxial rays we assume that the interface between the two liquids represents a thin lens that has no physical extension in the corresponding $3 \times 3$ ray-transfer matrix. The radius of curvature $R$ of the liquid interface depends on the common voltage $V_{A F}$ and the tilt $\theta_{y}$ in y-direction $\left(\theta_{x}=0\right)$ of the interface is controlled by the differential voltage between the two electrodes, $V_{y^{+}}$

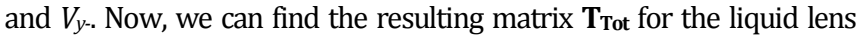
given as:

$$
\mathbf{T}_{\text {Tot }}=\mathbf{T}_{\text {ir }}\left(\begin{array}{ccc}
1 & 0 & 0 \\
\frac{n_{c}-n_{o}}{n_{o} R} & \frac{n_{c}}{n_{o}} & \left(\frac{n_{c}}{n_{o}}-1\right) \theta_{y} \\
0 & 0 & 1
\end{array}\right) \mathbf{T}_{\mathrm{fi}} .
$$

Thus, the resulting ray-transfer matrix can be written as:

$$
\mathbf{T}_{\text {Tot }}\left(\theta_{y}\right)=\left(\begin{array}{c}
1+\frac{\Delta n}{R} C_{o}-\frac{2 \mathrm{i} n_{a}}{k \sigma_{f}^{2}} C_{m} \\
\frac{\Delta n}{n_{a} R}-\frac{4 n_{a}}{k^{2} \sigma_{r}^{2} \sigma_{f}^{2}} C_{m}-\frac{2 \mathrm{i}}{k}\left(\frac{1}{\sigma_{f}^{2}}+\frac{1}{\sigma_{r}^{2}}+\frac{\Delta n}{R}\left(\frac{C_{c}}{\sigma_{f}^{2}}+\frac{C_{o}}{\sigma_{r}^{2}}\right)\right. \\
0
\end{array}\right.
$$

where

$$
\begin{aligned}
& C_{m}=C_{o}+C_{c}+\frac{\Delta n}{R} C_{o} C_{c} . \\
& \Delta n=n_{c}-n_{o}
\end{aligned}
$$

A simple way to implement a high-quality projector, which can scan a light pattern, is to combine the liquid lens with a commercial projector lens. If we apply the appropriate common voltage $V_{A F}$ to the liquid lens to let $R \rightarrow \infty$, the commercial projector lens is responsible for the imaging quality. The tilting feature of the liquid lens will make it appear as two opposing wedges with different refractive indexes, but the same, variable top angle. All other interfaces except the interface between the two wedges are normal to the optical axis. Therefore, in the paraxial approximation, light is deflected only due to the top angle of the wedges. Thus, depending on the differential voltage, the liquid lens will deflect the direction of light propagating through the projector. To demonstrate the application of the liquid lens with our raytracing model, we substitute the real projector lens system with a single thin lens. The liquid lens is placed against the projector lens and the aperture of the liquid lens defines the aperture of the imaging system. The distance from the lens to the object and image plane are $s_{1}$ and $s_{2}$, respectively. The imaging system is arranged for a given magnification, $M$, which means that $s_{1}=f(1+1 /|M|)$ and $s_{2}=f(1+|M|)$. A mask with the desired pattern is mounted in the object plane. When illuminating the mask from the back, the pattern is imaged onto a 3D object, which is located in the image plane. By inserting Eq.8 into the ray-transfer matrix for the imaging system we find: where $\Delta$ adjust for the effective thickness of the lens. The result is rather lengthy thus we will not present it in full length here. However, the four ABCD elements in the resulting matrix $\mathbf{T}_{\mathbf{C o m b}}$ are not affected by the tilting angle $\left(\theta_{y}\right)$. They will describe an imaging system based on the thin lens and merely see the liquid lens $(R \rightarrow \infty)$ as a thin flat window with a clearance radius of $\sigma_{r}$ inserted next to the lens. The elements, $E$ and $F$ in the resulting matrix depend on the tilting angle $\left(\theta_{y}\right)$ of the liquid interface. Element $E_{C o m b}$ specifies the positional offset of an imaged position in the image plane as a function of the tilt $\left(\theta_{y}\right)$ of the liquid interface. For $n_{a}=1$ we find:

$$
E_{\text {Comb }}\left(\theta_{y}\right)=\Delta n\left[f(1+|M|)+C_{o}-2 \mathrm{i} \frac{f(1+|M|)}{k \sigma_{r}^{2}} C_{o}\right] \theta_{y} \text {. }
$$

Element $F_{\text {Comb }}$ specifies the angular offset of an incoming ray in the image plane as a function of the tilt $\left(\theta_{y}\right)$ of the liquid interface:

$$
F_{\text {Comb }}\left(\theta_{y}\right)=\Delta n\left(1-2 \mathrm{i} \frac{C_{o}}{k \sigma_{r}^{2}}\right) \theta_{y} .
$$

The imaginary parts, which are responsible for diffraction, can be ignored for the setup we will present in the next section, and further $C_{o}$, $C_{c}, C_{m} \ll f(1+|M|)$. From Eq.11 it is clear that the y-position of a point or a line in the mask (object plane) can be shifted a distance of $\Delta y=$ $\Delta n f(1+\mid M) \theta_{y}$ in the image plane by applying a tilt of $\theta_{y}$ to the liquid interface in the liquid lens, given this setup.

\section{Experiments}

The experimental work consists of two parts. In the first part, we test the performance of the liquid lens when combined with a projector lens. The liquid lens and the projector lens are parts of a LED projector, which projects and scans a regular line pattern across a sensor placed in the image plane. In the second setup, the projector is inserted into a real dental scanner (3Shape), to demonstrate its ability to facilitate 3-D imaging and provide a measure of the accuracy obtained using this scanning projector. This setup is described in section 5 .

In Fig. 2, the schematic of the first experimental part is illustrated. The mask contains 27 parallel transparent lines evenly distributed throughout an area of $4.6 \times 4.6 \mathrm{~mm}$. The line spacing is $0.177 \mathrm{~mm}$, and the line width is $10 \mu \mathrm{m}$. 


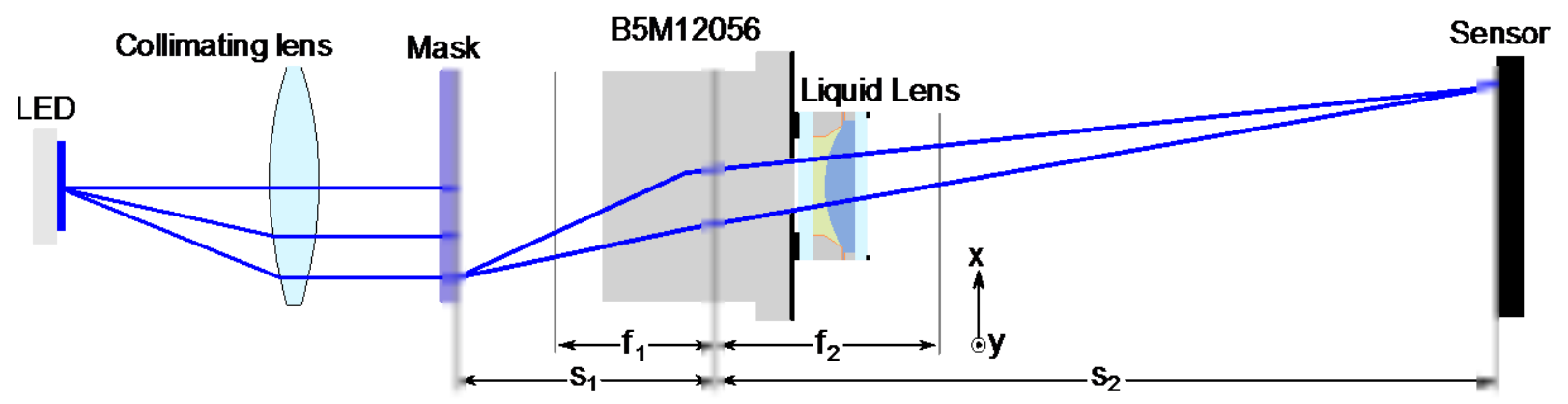

Fig. 2. The schematics for testing the general performance of the liquid lens, when combined with a projector lens.

The light from a blue LED source $(448 \mathrm{~nm})$ is guided to the mask via a condenser lens, and a projector lens images the mask onto the sensor. The sensor is a CMOS camera with a bare chip - no camera lens. The CMOS camera has $1920 \times 1200$ pixels with a pixel size of 5.86 $\times 5.86 \mu \mathrm{m}$. The camera is a black and white camera with global shutter, which can acquire images at a maximum frame rate of 162 frames per second. The distance between the projector lens and the sensor is set to $s_{2}=190 \mathrm{~mm}$, according to the working distance of the projector used in the 3Shape scanner. The liquid lens is combined with the projector lens B5M12056 (Lensagon). This lens system includes 5 lenses with an effective focal length of $f=12.0 \mathrm{~mm}$ and an exit aperture of $2.04 \mathrm{~mm} \emptyset$. The lens is designed in such a way that the Fourier plane of the object almost coincides with the exit aperture of the lens system. Therefore, we can mount the liquid lens directly against the exit aperture of the projector lens without changing the projector lens and without introducing any additional vignetting to the optical system. Given the working distance of the camera and its focal length, we can estimate the magnification of the imaging system to $M=-15.8$. The mask does not use the entire field of view of the projector lens. The angular field of view requested to image the diagonals of the mask is of $\pm 15.2^{\circ}$, only.

Table. 1. Parameters for the Baltic 617 lens [9], using an optical wavelength of $448 \mathrm{~nm}$.

\begin{tabular}{ccccc}
\hline Component & Type & $\begin{array}{c}\text { Refractive } \\
\text { index } \\
@ \text { 448 nm }\end{array}$ & $\begin{array}{c}\text { Clear. } \\
\text { radius } \\
\text { mm }\end{array}$ & $\begin{array}{c}\text { Mat. } \\
\text { thickness } \\
\text { mm }\end{array}$ \\
\hline Front apert. & Circular & - & $\sigma r=1.7$ & - \\
Front glass & D263T & $n_{g}=1.523$ & $>\sigma_{r}$ & $t_{f g}=0.3$ \\
Liquid 1 & Conduct. & $n_{c}=1.396$ & $>\sigma_{r}$ & $\mathrm{u}$ \\
Liquid 2 & Oil & $n_{o}=1.517$ & $>\sigma_{r}$ & $t_{i q}-\mathrm{u}$ \\
Rear glass & D263T & $n_{g}=1.523$ & $>\sigma_{r}$ & $t_{r g}=0.4$ \\
Rear apert. & Circular & - & $\sigma_{r}=1.2$ & - \\
\hline
\end{tabular}

For the blue LED, the relevant data for the liquid lens are listed in Tab. 1 . Here tiq $=1.15 \mathrm{~mm}$. The radius of curvature $R$ and thickness $u$ of the conductive liquid depend on the common voltage $V_{A F}$ In Fig. 3 , the focal length of the liquid lens is plotted as a function of $V_{A F}$. The focal length can be found as $f_{\text {liq }}=-1 / C_{\text {Tot }}(R)$ by inserting typical $R$ values listed in the data sheet [9] as a function of $V_{A F}$, and the refractive indexes listed in Tab. 1. Then, from Fig.3 we find that a common voltage $V_{A F}=$ $53 V_{\mathrm{RMS}}$ provides the infinite curvature of the membrane $(R \rightarrow \pm \infty)$ we sought for the implementation with the projector. According to the data sheet [9], we find that $u=0.564 \mathrm{~mm}$ at $V_{A F}=52.7 \mathrm{~V}$.

The liquid lens is interfaced to a PC via a driver chip. The driver generates the required voltages with 10 bit ADCs. Thus, the responses of the adjustable focus and tilt will be discrete. Each step will correspond to a step in voltage of $44.5 \mathrm{mV}$, with a total of 450 steps within the maximum voltage rating of $\pm 10.0 \mathrm{~V}$.

Fig. 4 illustrates an image of the line pattern incident on the sensor placed in the image plane. To analyze images captured during the experiments described below, three key metrics are computed: the mean intensity, the mean line position and the width of the projected line. The mean intensity of an image is used as a measure of vignetting. It may be computed as:

$$
\langle I(r, c)\rangle_{\text {image }}=\frac{1}{R_{i} C_{i}} \sum_{r=1}^{R_{i}} \sum_{c=1}^{C_{i}} I(r, c),
$$

where $I(r, c)$ is the intensity of the pixel at position $(r, c)$, and $R_{i}$ and $C_{i}$ are the number of rows and columns for the entire image, respectively. After the mean intensity has been found, the image is normalized before further processing.

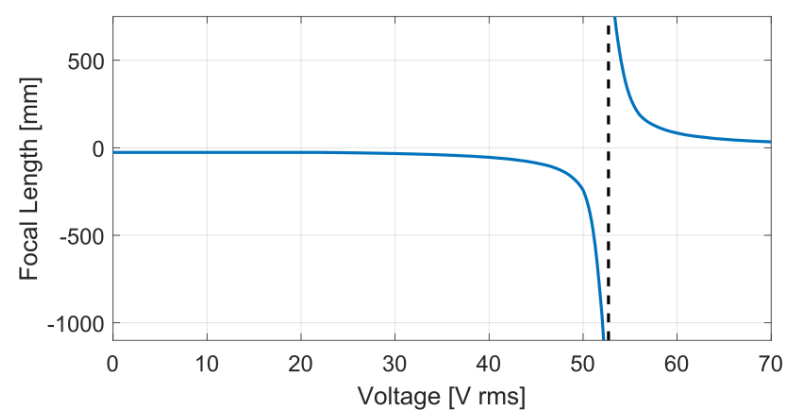

Fig. 3. The focal length $f_{\text {liq }}$ of the liquid lens is plotted as a function of the common voltage $V_{A F}$.

The line width calculation is sensitive to any tilt, distortion or bending of the lines. We have not experienced distortion, but the lines in the image are corrected for any tilt, using a Principal Axis Transform (PAT) [17].
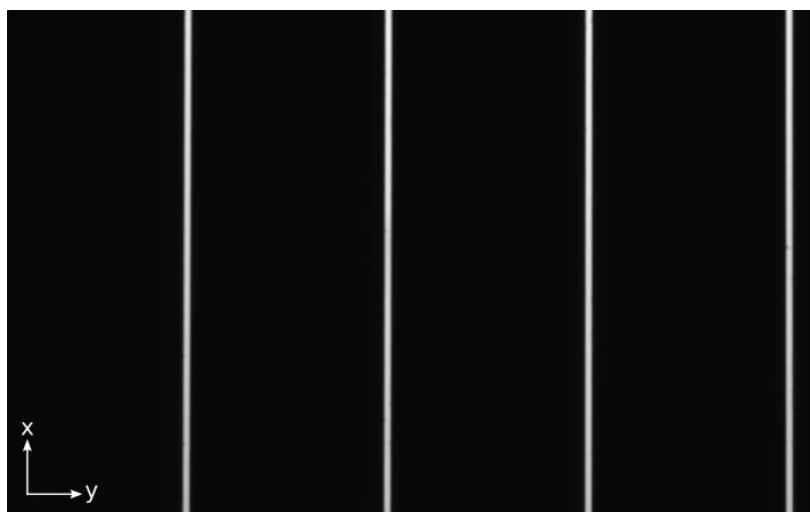

Fig. 4. The image illustrates a line pattern acquired by the sensor.

The principal axis transform finds the two principal axes in an image, i.e. orthogonal vectors that describe the variance of the data in decreasing order. Due to the nature of the images, the first principal axis will be exactly parallel to the projected line pattern, and may thus 
be used to find a transformation that maps the captured lines to perfectly vertical lines.

Once an image has been rotated according to the computed PAT, all the pixels are projected (summed up) on to a single horizontal row, having the intensity $I_{\text {line }}(c)$ :

$$
I_{\text {line }}(c)=\sum_{r=1}^{R_{i}} I(r, c),
$$

This dimensionality reduction greatly simplifies the handling of the data. To lessen noise, and to allow for the determination of line positions with sub-pixel accuracy, the data is convolved with a Gaussian window with a width of 30 points, and resampled at a factor of 1:10 using a low-pass filter. The center of each line may then be found as the maxima of the resulting line profile. The mean value of the line centers estimated for all the lines in a given image is used as a measure of the position of the projected line pattern. To describe the goodness of focus in a given image, the width of the centermost line is considered. The line width is determined as the Full Width at Half Maximum (FWHM) value of the resulting line profile. This provides a suitable way to compare focus across experiments and images. An image with good focus will exhibit a very small line width, whereas an image with poor focus will yield wider lines due to smearing of the scene.

\section{Results}

To quantify the behavior of the optical system when only tilt is applied to the liquid interface $(R \rightarrow \infty)$, the experimental setup described above was utilized. By visiting each discrete tilt step a multiple of times, a "swipe" of the line pattern using the liquid lens may be characterized. Fig. 5 shows the position of the central line pattern relative to the left-most position as a function of the individual steps in differential voltage $(44.5 \mathrm{mV})$ The left- and right-most positions are reached by applying a differential voltage of $10 V_{\mathrm{RMS}}$ and $-10 \mathrm{~V}_{\mathrm{RMS}}$ to electrode $\operatorname{set}\left(V_{y^{+}}, V_{y^{-}}\right)$.

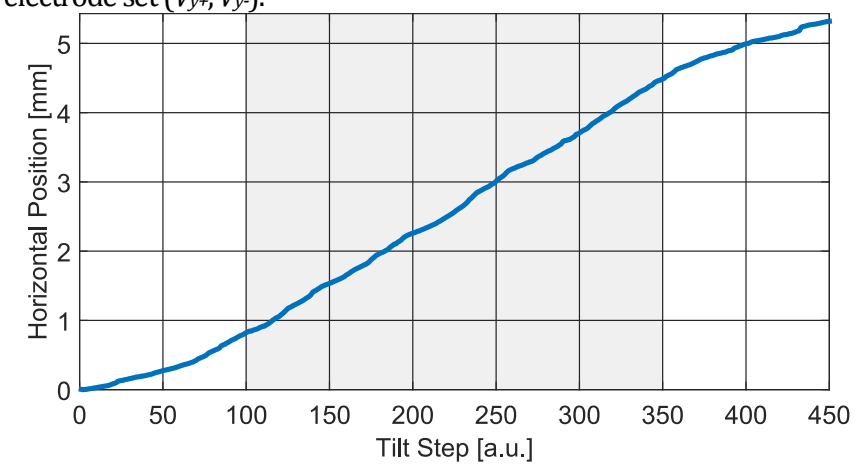

Fig. 5. Mean line position as a result of tilt. The grey area denotes the linear region.

As illustrated in Fig. 5, the position of the line pattern moves approximately linearly with the number of tilt steps. In the linear region from step 100 to step $350\left(\Delta V_{\mathrm{y}}= \pm 5.56 \mathrm{~V}_{\mathrm{RMS}}\right)$, denoted by a grey background in the figure, the curve follows an overall linear trajectory with some minor deviations, which never the less are reproducible.

Eq.1 may be rewritten to express the differential voltage that, when applied to a set of electrodes, results in a certain tilt of light rays, e.g. in the $y$-direction:

$$
\Delta V_{y, \max }=\left(V_{y+}-V_{y-}\right)_{\max }=2 G_{t i l t} \alpha_{y, \max } .
$$

We measure a total shift in position of $3.8 \mathrm{~mm}$ throughout the linear range $\left(\Delta V_{\mathrm{y}, \max }= \pm 5.56 \mathrm{~V}_{\mathrm{RMS}}\right)$, find a corresponding deflection of the beam by the liquid lens of $\alpha_{y}, \max = \pm 0.6^{\circ}$, and finally, using Eq.11 we find the corresponding tilt of the membrane of $\theta_{y, \max }= \pm 4.7^{\circ}$. Inserting the corresponding values of tilt and voltage into Eq.15, we find $G_{\text {tilt }}=9.3$ $\mathrm{V}_{\mathrm{RMS}} / \mathrm{deg}$. The values obtained for maximum light-ray tilt, $\alpha_{\text {max }}$, and the coefficient for Eq.15, $G_{\text {tilt, }}$ do agree with the values specified in the datasheet [9].

Fig. 6 shows the result of fitting a straight line to the points in the linear region, and subsequently calculating the deviation of points from the ideal linear trajectory. The standard deviation from a perfect linear response is $26.3 \mu \mathrm{m}$, while the reproducibility is better than a few microns. In the nonlinear regions below step 100 and above step 350, denoted by a white background, the response saturates slightly.

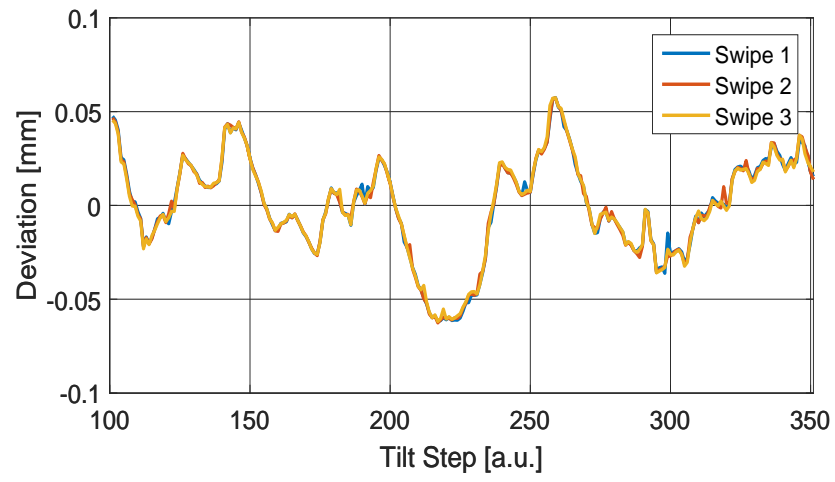

Fig. 6. Deviation from linearity of points in the nearly-linear region.

To investigate the presence of cross-talk between focus and tilt, the width of the centermost line in each picture is found for each discrete step of tilt. The resulting curve for the measured linewidth is shown in Fig. 7. As can be seen in the figure, the linewidth has its minimum at tilt step 225, where no tilt is applied and where the optical setup provides an image of the line pattern in focus $(R \rightarrow \infty)$. When, the liquid interface is tilted in either direction, the linewidth increases as a result of defocus and optical aberrations. The broadening of the linewidth can, to some extent, be reduced by adjusting the focus $(R)$ of the liquid lens, but not completely. However, by constraining the tilt to within the boundaries of the linear region, the effect of cross-talk will be less pronounced, and the broadening of the line width will remain within $30 \%$ of its minimum value of $0.13 \mathrm{~mm}$.

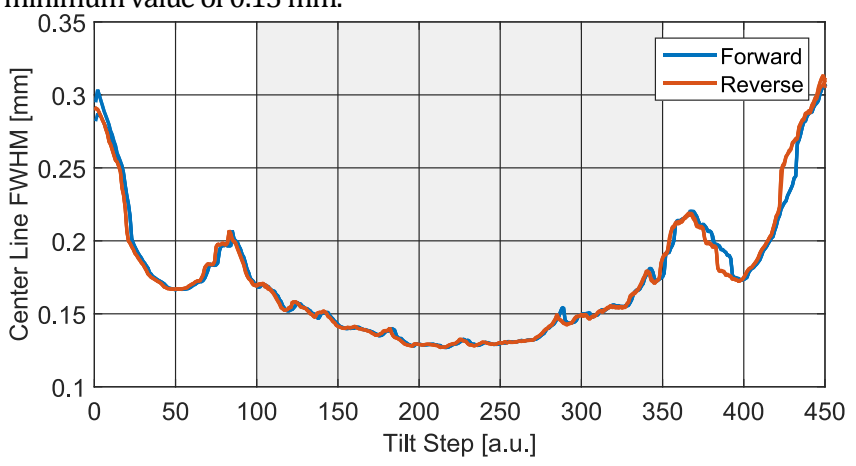

Fig. 7. Line width of centermost captured line as function of tilt.

During the cross-talk experiment discussed above, it was observed that positions reached by movement in one direction were offset compared to positions reached by movement in the opposite direction. To further investigate the presence of hysteresis, an experiment was conducted where repeated movements to the relaxed position, i.e. the position corresponding to $0^{\circ}$ tilt, from positions to either side $\left(\alpha_{y}=0.6^{\circ}\right.$ and $\alpha_{y}=-0.6^{\circ}$ ) were conducted. The distribution of positions resulting from several repetitions of these movements is shown in Fig. 8. As it is evident in the figure, targeting the same position from both directions 
will not result in the same projection of the light pattern. For unknown reasons, the spread of the distribution for the relaxed positions when moving from left side $\left(\alpha_{y}=-0.6^{\circ}\right)$ is smaller than when moving from the right side $\left(\alpha_{y}=0.6^{\circ}\right)$. Thus, the hysteresis is significant, but it can be accounted for by attempting precise changes of tilt in a single direction only, and by starting any positioning of the tilt from the corresponding extreme reference tilt position at either $\alpha_{y}= \pm 0.6^{\circ}$.

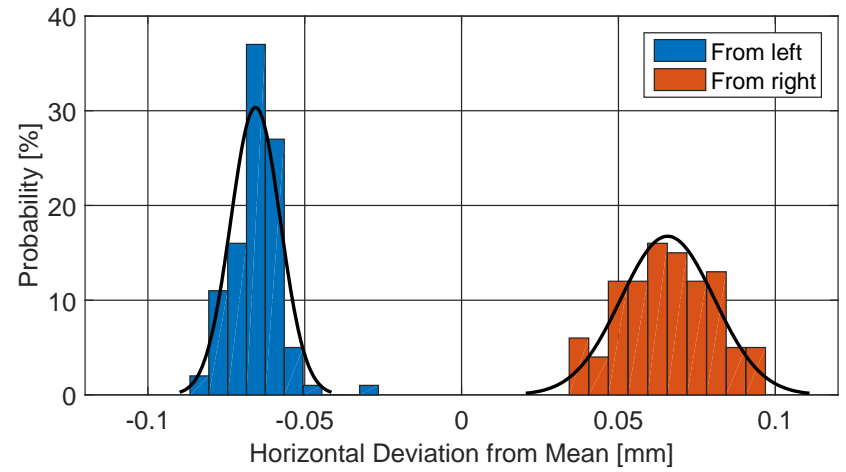

Fig. 8. Distribution of positions reached when centering by tilt from a position being either left $\left(\alpha_{y}=-0.6^{\circ}\right)$ or right $\left(\alpha_{y}=0.6^{\circ}\right)$ of the center.

Finally, in order to gauge the speed of the system, a series of steps were recorded using the highest frame rate of the camera (162 f/s) and repeated in order to obtain descriptive trajectories of the system. Fig. 9 shows changes in position caused by steps of varying sizes. The positions have been normalized to allow for comparisons between the different trajectories.

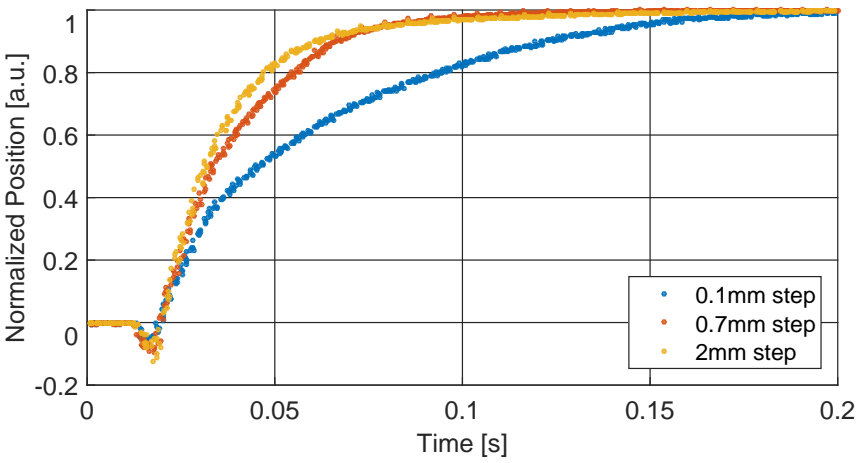

Fig. 9. Temporal response of liquid lens for steps of varying magnitude.

In all cases, the trajectory starts by peaking at a negative position. This is most likely caused by the inertia of the system, coupled with the dynamics of the liquids. Thereafter, all the curves exhibit positive responses, which follow exponential decays characterized by different time constants. After 50-120 ms, the system stabilizes at its new tilt. Interestingly, large steps are executed faster than small steps.

\section{Application}

To demonstrate the functionality of the liquid lens in the area of 3D scanning, the projector and the liquid lens are incorporated into a real dental 3D imaging scanner (3Shape). For comparison, this experimental scanner is tested back-to-back with a commercial 3D imaging scanner (D900L, 3Shape [18]), while measuring on a test piece and a real dental cast.

Fig. 10 illustrates the schematics of the scanner. The projector is mounted and centered on the upper horizontal bar together with four cameras. In this setup, the cameras acquire images simultaneously from four different directions, and thus provide an overdetermined stereo vision of the subject for each step of the scanner. The liquid lens steps the projected line pattern across the subject in angular steps of $0.0036^{\circ}$. After a complete scan by the liquid lens, the subject is rotated by an angle of $60^{\circ}$. in the horizontal plane, and a new complete scan is initiated. After a single loop, most occlusions will have been eliminated - even for complex subjects, such as a dental cast.

Before the scanning starts, the projector and cameras are calibrated using a target designed for the purpose. The target consists of a flat glass plate coated with a diffuse white coating and a $25 \times 26$ black chrome dot matrix printed on top. Each dot has a radius of $0.5 \mathrm{~mm}$, and the dots are spaced $2 \mathrm{~mm}$ apart. During calibration, the target is rotated to various angular positions with respect to the rotation axis (see Fig. 10). Here, series of 2D images of the dot matrix are captured with each camera. From each image, the dot center positions are tracked with sub-pixel accuracy in the software and used to generate software models for each camera. The projector lines are subsequently imaged onto the same target, and acquired 2D camera images of the lines are used to make a software model for the line projector. The software model is required to calculate transformations from the projector, including the liquid lens to the subject.

In order to assess the performance of the experimental liquid lens scanner, a precisely machined gauge block is scanned using the production scanner and the experimental scanner. For each point cloud produced by a scanner, points near each of the two large sides of the gauge block are used to fit two planes, one for each side. These two planes may then be used for characterizing the quality of a given scan. The two large sides of the gauge block are known to be exactly 15.000 $\mathrm{mm}$ apart. Thus, the distance between two fitted planes compared to the true distance yields a measure of the overall precision of a scan. In Tab. 2, the deviation in measured distance from the true distance is called the Error.

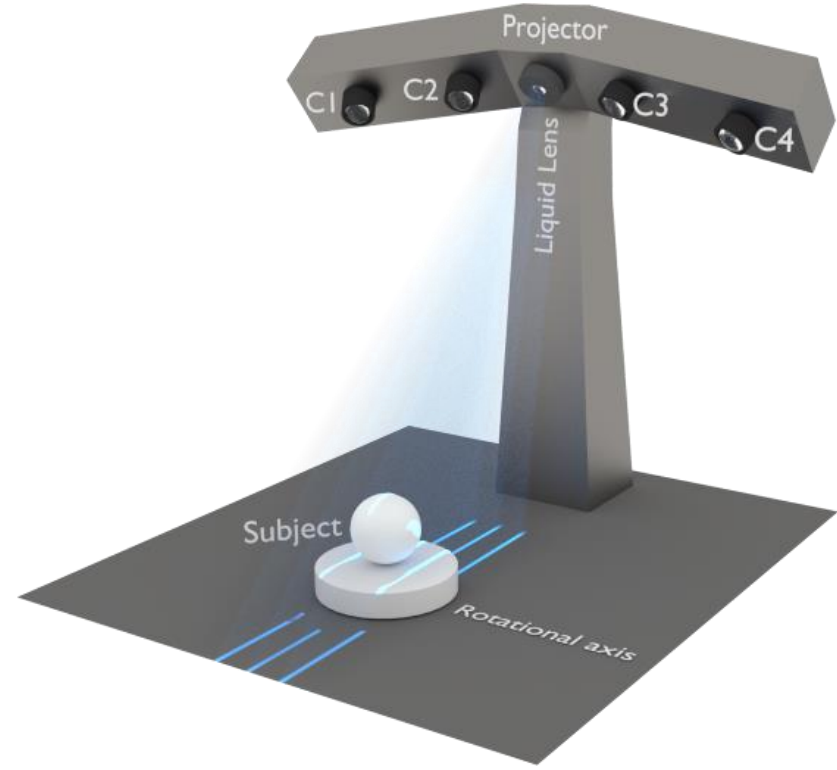

Fig. 10 Simplified 3D rendering of the experimental liquid lens scanner. The projector with the liquid lens is centered on the horizontal bar with the four cameras, $\mathrm{C} 1, \mathrm{C} 2, \mathrm{C} 3$ and C4.

The standard deviation of points associated with a plane, to that particular plane is calculated. In Tab. 2, this measure is called the Noise. Finally, the variability of points can be examined by computing the percentage of points associated with a plane that lie within $20 \mu \mathrm{m}$ of that plane. This metric serves to indicate whether a scanner's imprecision is caused by a general lack of accuracy, or by one or more specific sources of error, e.g. edges. 
Tab. 2 shows a comparison of the gauge block metrics obtained from point clouds produced by the production scanner and the experimental scanner. It is immediately clear that the experimental scanner produces far more points than the production scanner. This is simply due to data reduction optimizations such as point skipping, which are only activated in the production scanner. As such, the difference in the number of data points does not influence the actual precision of either scanner.

Table. 2. Comparison of gauge block point cloud metrics using production and experimental scanners.

\begin{tabular}{lcc}
\hline & Prod. scanner & Liquid lens \\
& (D2000, 3Shape) & scanner \\
\hline Points & 28275 & 66273 \\
Distance $[\mathrm{mm}]$ & 15.0129 & 14.9731 \\
Error $[\mu \mathrm{m}]$ & 12.89 & 26.86 \\
Noise $[\mu \mathrm{m}]$ & 6.2 & 6.73 \\
Points within $20 \mu \mathrm{m}[\%]$ & 92.11 & 81.72 \\
\hline
\end{tabular}

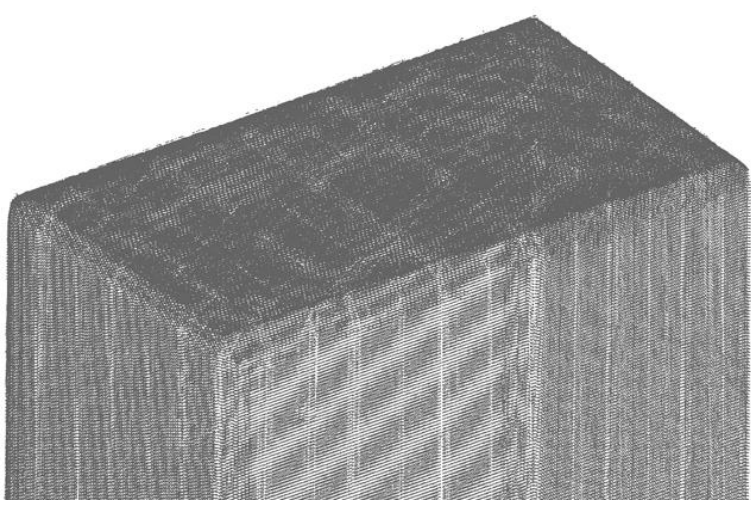

Fig. 11. Point cloud representation of gauge block produced by the experimental scanner.

When comparing the remaining metrics, it is evident that the production scanner attains a higher level of precision throughout. However, when considering the assumptions and simplifications used to realize the prototype liquid lens scanner, the performance is indeed satisfying. The liquid lens scanner exhibits an error twice that of the production scanner, with a comparable noise magnitude.

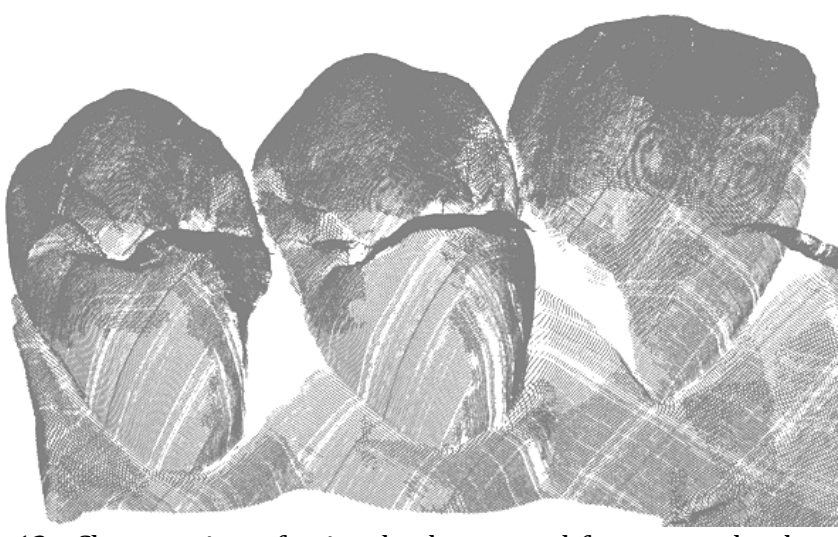

Fig. 12. Close-up view of point cloud generated from a cast by the experimental scanner.

Fig. 11 shows a portion of the point cloud produced by the liquid lens scanner, when scanning the gauge block described above. As it is evident from the figure, the scanner is capable of providing close to full coverage of the object being scanned.
As shown in Fig.6, the liquid lens exhibits a nonlinear trajectory when swiping through the range of tilt steps. However, the software model used for the liquid lens relies on linear trajectory in order to calculate transformations from the projector to the subject. Thus, using a nonlinear model would provide a more accurate description of the behavior of the liquid lens. However, in order to simplify the software implementation of the scanner, a simple piece-wise linear function was used instead. This model was sufficiently accurate for a majority of the tilt steps, allowing the capture of high quality measurements. Nevertheless, some tilt steps deviated so far from linearity that they were excluded by the scanner's matching algorithm. Points associated with these tilt steps appear as bands of missing information in Fig.11 and Fig.12.

Fig. 12 and Fig. 13 showcase the results obtained when scanning a real cast. Fig. 12 depicts a close-up crop of the point cloud produced by the experimental scanner when scanning the aforementioned cast. As evident in the figure, the scanner is capable of producing high resolution scans, even in the face of complex geometry with many occlusions.

Fig. 13 shows the final 3D model, once a proprietary 3Shape meshing algorithm has been applied to the point cloud discussed above. The meshing algorithm attempts to close any holes in the point cloud caused by occlusions or areas of high reflectivity. The end result is a very high fidelity 3D model that can be used by dental professionals.

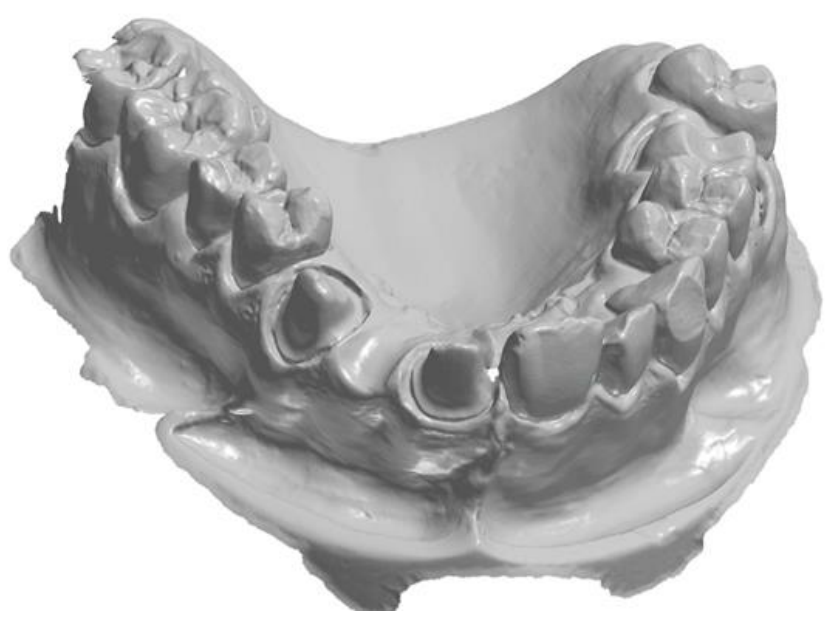

Fig. 13. Final 3D model after processing by 3Shape's software.

\section{Discussion}

We found from our results that the liquid lens is capable of translating the pattern of light a total of $5.5 \mathrm{~mm}$ at a working distance of $190 \mathrm{~cm}$. This corresponds to an angular deflection of $1.66^{\circ}$. If only the approximately linear region is considered, the liquid lens is still capable of a translation of $3.8 \mathrm{~mm}$ or $1.15^{\circ}$.

In the linear region of the tilt response (Fig. 5), minor deviations were discovered (Fig. 6). These could, to some extent, be corrected for by using ADCs with a higher resolution than 10 bit, and by using a lookup table for the relation between the positions and the voltages. Alternatively, a more precise and reliable mathematical model of the liquid lens to calculate the transforms from projector to subject could be determined. This could be achieved by capturing images of the projected scan lines on a known surface, i.e. the dot matrix plate, whilst altering the tilt of the liquid lens.

The results of e.g. Eq.11 and Eq.12 rely on the assumption that the rays involved in the imaging process of the projector all obey the paraxial assumption. Therefore, we expect the model be valid for 
central areas of the image (within $\pm 5^{\circ}$ of angular field of view), and within the relevant range of tilt $\left( \pm 5^{\circ}\right)$ of the membrane as a function of voltage. However, the paraxial approximation will not be valid for the marginal rays describing the field of view of the mask. The marginal angles given the projector lens and the size of the mask are $\pm 15.2^{\circ}$.

Consider a non-paraxial model for two thin wedges with different refractive index, $n_{c}$ and $n_{o}$, but with the same top-angle, $\theta$. Put together, they form a rectangular component, where the front and rear facets are normal to the optical axis. The normal of the shared surface will then be tilted $\theta$ relative to the optical axis. See Fig. 14 .

We find the following non-paraxial deflection $\delta$ for a ray incident at an angle of $\alpha_{1}$ at the front facet of the double wedge given as:
At Fig. 7, we argued that defocus was a part of the broadening of the line width. And we observed that the broadening could be reduced partly by adjusting the focus. Similarly, by adjusting the focal length during 3D-image acquisitions higher quality scans of individual objects could be achieved, allowing for a better depth resolution. This would be accomplished by capturing images of an object while changing the focal length iteratively, and afterwards stitching together the parts of an image that define the individual scanlines best. This could further open up for applications involving large objects, given the flexible working distance, and by eventually introducing the liquid lenses with adjustable focus in the camera lenses as well. As long as such adjustments of the focal length are small compared to the working

$$
\delta=\sin ^{-1}\left(\frac{n_{o}}{n_{a}} \sin \left(\sin ^{-1}\left(\frac{n_{c}}{n_{o}} \sin \left(\sin ^{-1}\left(\frac{n_{a}}{n_{c}} \sin (\theta)\right)+\theta\right)\right)-\theta\right)\right)-\alpha_{1} .
$$

In Fig. 14 the difference between the non-paraxial deflection (Eq.16) and the paraxial deflection (Eq.12) is plotted as a function of the angle $\theta$ at various angles of incidence. The paraxial deflection provides results similar to the non-paraxial deflection with an error of $1.2 \%$ of full beam deflection for $\left|\alpha_{1}\right|<5^{\circ}$ and $-5^{\circ}>\theta>5^{\circ}$. Thus, as expected, the model we proposed in section 2 is valid provided that the paraxial assumption is obeyed for the rays. In case of $\left|\alpha_{1}\right|>5^{\circ}$ and $\theta \neq 0$, we are beyond the paraxial approximation for the projector lens and the non-paraxial deflection in the two wedges introduces a significant deviation from the paraxial deflection of up to $7.1 \%$ of the full paraxial deflection within the range of interest of, $-5^{\circ}>\theta>5^{\circ}$. However, similar to the above discussion concerning the minor non-linear deviations in the voltage-tilt response, these non-paraxial deviations in line deflection can be corrected for by calibrating the entire scan of the line projector, and include them in the model for the line projector. In this specific case we expect that the non-paraxial deviations to some extend has been caught by the post processing, due to the redundancy of capturing data from several angular positions of the object. Therefore, the non-paraxial deviations pose limitations to our ray-tracing model (Eq.11 and Eq.12), but not necessarily the application itself.

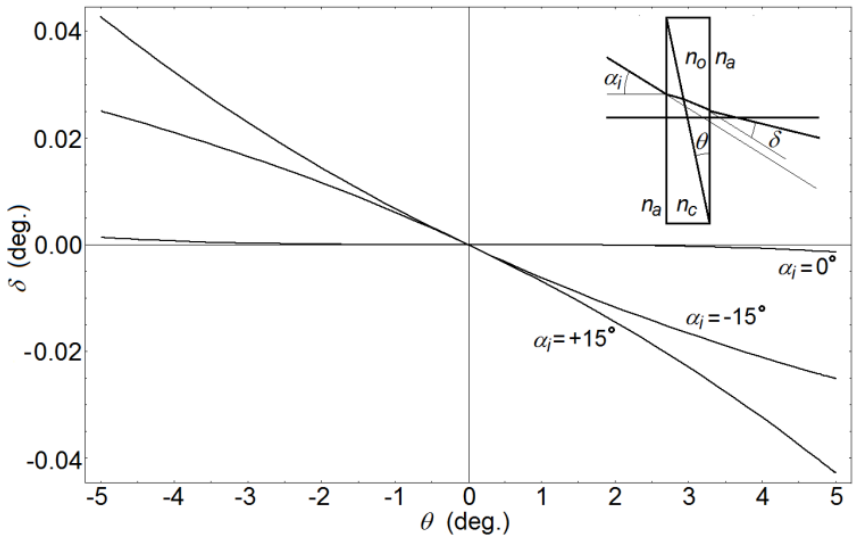

Fig. 14. The insert illustrates the two wedges. The curves illustrate the non-paraxial deflections as function of $\theta$ at various angles of incidence on the wedges.

In this work we have only addressed the characteristics of the liquid lens in the specific case where the focal length is very large $(R \rightarrow \infty)$. We observed that when the membrane is adjusted to a focal length of few $\mathrm{mm}$, the range of tilt is significantly reduced. Similarly, it is to be expected that most of our characteristics such as e.g. the cross talk in Fig. 7 will change if $R$ is set to a finite value. distance, the characteristics provided by this work will be valid.

The datasheet illustrates the thermal dependences on the parameters, affecting the relation between the curvature of the membrane and the common voltage, $G_{\text {tilt, }}$ and their response times. The operating temperature ranges from $-10^{\circ} \mathrm{C}$ to $+60^{\circ} \mathrm{C}$. The value of $G_{\text {tilt }}$ increases almost linearly with temperature by $0.4 \%$ per ${ }^{\circ} \mathrm{C}$. The time response for tilting the membrane decreases by a factor of 3-4 as the temperature increases from $-10^{\circ} \mathrm{C}$ to $+60^{\circ} \mathrm{C}$. The relation between membrane curvature and common voltage is described as linear, where the slope and the voltage offset depends slightly on temperature.

The slope decreases with temperature by $0.3 \%$ per ${ }^{\circ} \mathrm{C}$, while the voltage offset peaks at $20^{\circ} \mathrm{C}$ and otherwise varies by $0.1 \%$ per ${ }^{\circ} \mathrm{C}$. The corresponding time response depends on the curvature of the membrane. Within 0-1 diopter (large $R$-values) the time response is mostly constant versus temperature.

As detailed in Fig. 9, the liquid lens has a tilting response time of 50$120 \mathrm{~ms}$. Because of the high amount of computation required for aligning and merging images into 3D points, the liquid lens is not the bottleneck of the system in terms of scanning speed.

\section{Conclusion}

This paper has presented a liquid lens, integrated in a projector with the application of scanning the image of a line pattern transversely across an object. The liquid lens is based on the EWOD technology. Both the focal length and the tilt of the lens can be electrically adjusted. The performance of the projector has been characterized with the following results: Linearity of the scan is within $26.3 \mu \mathrm{m}$, when considering only the linear scanning range of $3.7 \mathrm{~mm}$. Crosstalk between the focus and tilt in combination with optical aberrations give a broadening of the central line width of less than $30 \%$ its minimum width. Hysteresis gives an offset of $\pm 0.07 \mathrm{~mm}$ at zero tilt when approaching from the respective maximum tilt values $\left(\alpha= \pm 0.6^{\circ}\right)$. For responses above $120 \mathrm{~ms}$, we observe no effects of the inertia of the liquids in the lens. Finally, the projector has been integrated into a 3D imaging scanner and has been tested back-to-back with a commercial 3D imaging scanner (3Shape) on a test piece. The error on measuring a distance between two facets of the test piece with the system, based on the scanning projector, was $27 \mu \mathrm{m}$ out of $15.000 \mathrm{~mm}$. The difference in errors obtained by the two systems differed by a factor of only two.

\section{Acknowledgement}

The work has been carried out in cooperation with 3Shape and components have been granted by Varioptic for experimentation. 


\section{References}

1. I. Moring, H. Ailisto, V. Koivunen and R. Myllyla, "Active 3-D vision systems for automatic model-based shape inspection," Opt. Lasers Eng. 10, 3-4 (1989).

2. G. Bickel, G. Hausler and M. Maul, "Triangulation with expanded range of depth," Opt. Eng. 24, 975.977 (1985).

3. Arroyo and C.A. Greated, "Stereoscopic particle image velocimetry", Meas. Sci. Technol. 2, 1161-1186 (1991).

4. J. Salvi, J. Pages and J. Batlle, "Pattern codification strategies in structured light systems", Pattern Recognition, 37(4), 827-849 (2004).

5. A. Dipanda and S. Woo, "Efficient correspondence problemsolving in $3 \mathrm{D}$ shape reconstruction using a structured light system", Optical Engineering, 44(9), 1-14 (2005).

6. J. Geng, "Structured-light 3D surface imaging: a tutorial", Advances in Optics and Photonics, 3, 128-160 (2011).

7. S. Huang, L. Xie, Z. Wang, Z. Zhang, F. Gao and X. Jiang, "Accurate projector calibration method by using an optical coaxial camera", Appl. Opt., 54, 789-795 (2015)

8. H.H. Refrai, J.J. Sluss, M.P. Tull, "Digital micromirror device for optical scanning applications", Optical Engineering 46, 085401 (2007).

9. Varioptic, "Baltic 617", Parrot SA Confidential (2014).

10. G. Kloos, Matrix Methods for Optical Layout, SPIE Vol. No.: TT77, 2007, ISBN: 9780819467805.

11. B. Berge and J. Peseux, "Variable focal lens controlled by an external voltage: An application of electrowetting," The European Physical Journal E3, 159-163 (2000).

12. R. Shamai, D. Andelman, B. Berge and R. Hayes, "Water, electricity, and between ... On electrowetting and its applications", Soft matters 4, 38. (2008).

13. S. Kuiper and B. H. W. Hendriks, "Variable-focus liquid lens for miniature cameras", Applied Physics Letters, 85, 1128-1130 (2004).

14. A. E. Siegman, Lasers, University, Science, 1986.

15. H.T. Yura and S.G. Hanson, "Optical beam wave propagation through complex optical systems", J. Opt. Soc. Am. A, 4, 19311948 (1987).

16. H.T. Yura and S.M. Beck,"Temporal averaging of atmospheric turbulence-induced optical scintillation," Optics Express 23, 22867-22882 (2015).

17. R.C. Gonzales and P Wintz, Digital Picture Processing, Reading, MA: Addison-Wesley, 1987, ISBN 0-201-11026-1.

18. 3Shape, Dental System ${ }^{\text {TM }}$ white paper, 2015. 